\title{
Societies and change
}

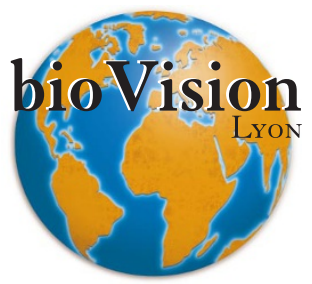

\author{
Axel Kahn and Heinz Imhof
}

The remarkable species, Homo sapiens, is on the verge of another technical transition for which it needs to plan. At its core is genetics and molecular biology.

It would be churlish to pretend that genetic engineering and the possibilities that it raises are merely extensions of what humans have been doing for thousands of years. It is indeed the case that humankind has been manipulating life, including human life, at least since the dawn of agriculture. It is also true that few things in this world are now natural. Our environments certainly are not: the fact that most of Europe is not covered in ancient forest, for instance, is because earlier men and women cleared the land to rear animals and grow plants. Furthermore, it is inescapable that most of the changes to animals and plants made through breeding have been for human benefit and pleasure.

However, genetic engineering can only be said to be the same as what has gone before in that it is another process for change. It is another mechanism by which humans can change living organisms for their own ends. Genetic engineering is, of course, a rapid way of bringing about change, much faster than evolution, and faster, too, than "traditional" plant and animal breeding. But it is no more rapid than other forms of engineering such as those that have led to the development of the train, the airplane, the computer, or personal communication devices. All of these advances lead societies into fundamental changes. And yet none has elicited the same visceral reaction as genetic engineering.

What sets genetic engineering apart is that its clay is life itself. Life, many people feel, is sacrosanct and beyond human probing. If, as in modern societies, a holistic and interconnected view of nature is superimposed upon the holiness of life, then any intrusion into any life form becomes an abuse of human sanctity.

The other disquieting aspect of genetic engineering may be that it is largely imperceptible. Few people may realize that all insulin-dependent diabetics in the developed world are now treated with a human form of insulin produced through genetic engineer-

Axel Kahn is at the Institut Cochin de Génétique Moléculaire, INSERM Unit 129, 24 rue Faubourg St. Jacques, 75014 Paris, France (kahn@icgm.cochin.inserm.fr). HeinzImhof is chief executive officer of Novartis Seeds, $\mathrm{CH}$ 4002 Basel, Switzerland. ing. Twenty years ago, insulin for diabetics came from the organs of slaughtered pigs or cattle. Before genetic engineering, human growth hormone for treating dwarfism was extracted from the brains of dead people.

Whereas the stealth of genetic engineering in medicine seemed inoffensive, its appearance in transgenic food has been a cause of considerable perturbation, especially in Europe.

In some respects, the trauma of transgenic food is surprising. The tools for plant genetic engineering have been around since 1982. Even before then, techniques such as protoplast fusion and assisted pollination were being used to create crosses between plant species. The achievements of genetic engineering in agriculture are scientifically unspectacular.

What stands against current developments, however, is the perception that they bear the imprint of the ambitions of multinational companies. They do not, to many people's view, address any particularly pressing human need. One of the main planks of the rejection of agricultural genetic engineering in Europe is that it seeks immediate profits in developed countries without either a wellenunciated popular demand for the products, or a clear connection to broader, worldwide questions.

The other dimension to current attitudes toward genetic modification in foods has been a rise in the role of the consumer. Consumers demand that products work reliably. They demand safety, and they demand redress if something goes wrong. Increasingly, they further demand that businesses produce products in an ethical way. Sometimes they also demand that products are needed. The main issue is the lack of a trusted EU agency responsible for approving products containing genetically engineered organisms.

Consumers demand to know how agricultural innovations come about. This new demand stems, in part at least, from the shock of the epizootic outbreak of bovine spongiform encephalitis (BSE). BSE was not the outcome of a scientific development. It was the result of a misguided attempt to feed cattle more cheaply by feeding them the rendered remains of other animals. It was assumed that the high temperature rendering process would destroy any infectious agents. It did not. That error was compounded when scientific experts gave reassurances that the further passage of BSE to humans was unlikely. When a new variant of the causative organism of the human brain disease, CJD, emerged, public trust in "experts" evaporated and their interest in the production process was provoked.

Among this suspicion of science, on the one hand, and the mobilization of consumers on the other, the campaigns of those who are philosophically opposed to biotechnology have naturally found fertile ground. Interwoven with this have been anxieties about "manipulation of life itself" and comparisons between genetic engineering and recent consequences of eugenics.

Of course, there is much we do not know even about those products and processes that have been developed through scientific research. People who hold themselves up as experts — on science, technology, the environment, social policy, culture, or religion-need to be ruthlessly honest about what they do know and what they do not know. The science community has a particular obligation to provide public information: it needs to be active in ensuring that new information reaches public understanding.

Furnished with information, the public must exercise its rights seriously. The public has a duty to recognize that many (if not most) things in life-like the choice of a marriage partner, for instance-are decided without full information or absolute certainty. It must understand, too, that most decisions in real life have dimensions that go beyond simply "stop" or "go." Saying "no" to one kind of product or process means saying "yes" to the alternatives. In denying innovation, one should be conscious that one is accepting the status quo.

In general, the level of acceptable risk depends upon perceived needs. That means that people need to be clear on whose behalf it is they are acting or deciding. Knowledge may be universal but that does not mean scientists study all aspects of a problem. Large companies may be multinational but that does not mean they are truly global in their outlook. A person may indeed be a citizen of a town, of a country, and of the world. But that does not mean they understand the needs of each location equally.

In a peaceful, sanitary, agriculturally productive society, the most prominent deficiency, and thus the most acceptable use of new technology, may be in medicine. People in conflict-ridden regions where water is short and agriculture ravaged by diseases and pests may perceive things differently. 